\title{
The Precipitation and Dissolution of Alloy Iron Carbides in Vacuum Carburization Processes for Automotive and Aircraft Applications-Part II
}

\author{
Kula Piotr ${ }^{a}$, Wolowiec Emilia ${ }^{b}$, Pietrasik Robert ${ }^{c}$, Dybowski Konrad ${ }^{d}$, \\ Klimek Leszek ${ }^{\mathrm{e}}$
}

Institute of Materials Science and Engineering, Technical University of Lodz, Stefanowskiego 1/15, 90-924 Lodz, Poland

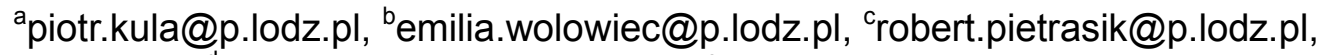
konrad.dybowski@p.lodz.pl, ${ }^{\mathrm{e}}$ leszek.klimek@p.lodz.pl

Keywords: Vacuum carburizing; carbides; heat treatment; neural networks

\begin{abstract}
The article is dedicated to the experiments and tests on the phenomena of precipitation and dissolution of alloy iron carbides in vacuum carburization processes. Special attention has been paid to the possibility of using artificial neural networks to predict the speed of the processes examined. In the section below, we are presenting the precipitation phenomena taking place in vacuum carburization processes and the experiments that were conducted. Moreover, a qualitative and metallographic analysis of carbide phenomena was described together with the method of numerical modelling and predicting the processes with the use of artificial neural networks.
\end{abstract}

\section{Introduction}

Rapid technical progress of civilization makes devising new technologies a multi-faceted issue. Nowadays, machine and software designers focus on simultaneously selecting construction characteristics and elements of machines, specifying their technological processing, optimal software and the most suitable materials. As a result it is possible to cater to human needs in the best of ways and at the lowest cost. New possibilities for designing machines and devices are offered by tools which support designer work, including those using artificial intelligence. The advantages and efficiency of those tools make them increasingly popular in this field [1-4].

This article gives an account of the research on an instrument for simulating vacuum carburizing of steel. Carburization itself is well-researched. However, research on the concomitant carbide forming processes, common for this type of processing, is not satisfactory.

Mathematical models of the phenomena of precipitation and dissolution of alloy carbides in austenite are difficult to build due to the complexity of the processes described above. Artificial neural networks do not require any mathematical and physical equations, because during the learning process, based on empirical cases, they formulate the relations between phenomenon parameters independently, and thereby they are considered a proper tool in modeling the problem discussed [5-8]. A correctly built network can map even very complex functions and is easy to use (in practice, it builds the necessary model according to the examples presented) [9]. It is highly probable that such a network can be used wherever there are problems connected with working out mathematical models, which provides an opportunity to construct models for barely known and examined phenomena and processes.

The use of neural networking has made it possible to skip the stage of creating a mathematical and physical model of the phenomenon (for which there is no account of exact mathematical relations to be found in literature) and directly pass on to the stage of simulating the material properties on the basis of carburization parameters.

The following subchapters briefly deal with the essence and purposes of vacuum carburization, the research on possible neural network uses and the architecture of a given neural network which is an actual application of the design. 


\section{Vacuum carburizing experiments}

Vacuum carburizing is one of the thermochemical processing methods [10,11]. Carburization consists in saturating steel with carbon at high temperatures. As a result, an appropriate carbon concentration profile is formed in the surface layer. Normally, it consists of the saturation stage during which atmospheric carbon is applied to a steel surface, and the diffusion stage - during which the carbon from the steel surface is distributed inside the steel element. The course of the process depends on the treated element (material type, dimensions, condition of the surface), the type and flow of the carbonizing atmosphere, the process temperature and pressure, as well as the duration of the saturation and diffusion stages. The purpose of carburizing is to impart certain mechanical, physical and chemical properties to steal, optimal for a given application. On the surface of the machine a protective layer is formed that is resistant to abrasive wear and contact fatigue, while preserving the necessary core ductility [12].

The main objective of material experiments was to observe successive stages of carbides forming and dissolving in the material in order to be able to work out, based on them, a set of training data for an artificial neural network. The material and thermal processing was described in the part I of this article as well as material research analysis.

\section{Numerical analysis}

The main aim of the material experiments was to examine the precipitation phenomena and design a set of training data for an artificial neural network. The most important task of a neural network was prediction, i.e. forecasting results for the data included in the problem domain, but outside the set of learning cases.

Based on the observations of the phenomenon and empirical data obtained during material experiments, a system of MLP-type neural networks (multi-layer one-direction sigmoid networks) was worked out [13-15]. The networks were taught with the use of the BFGS method (Broyden-Fletcher-Goldfarb-Shanno method) until satisfactory results were reached.

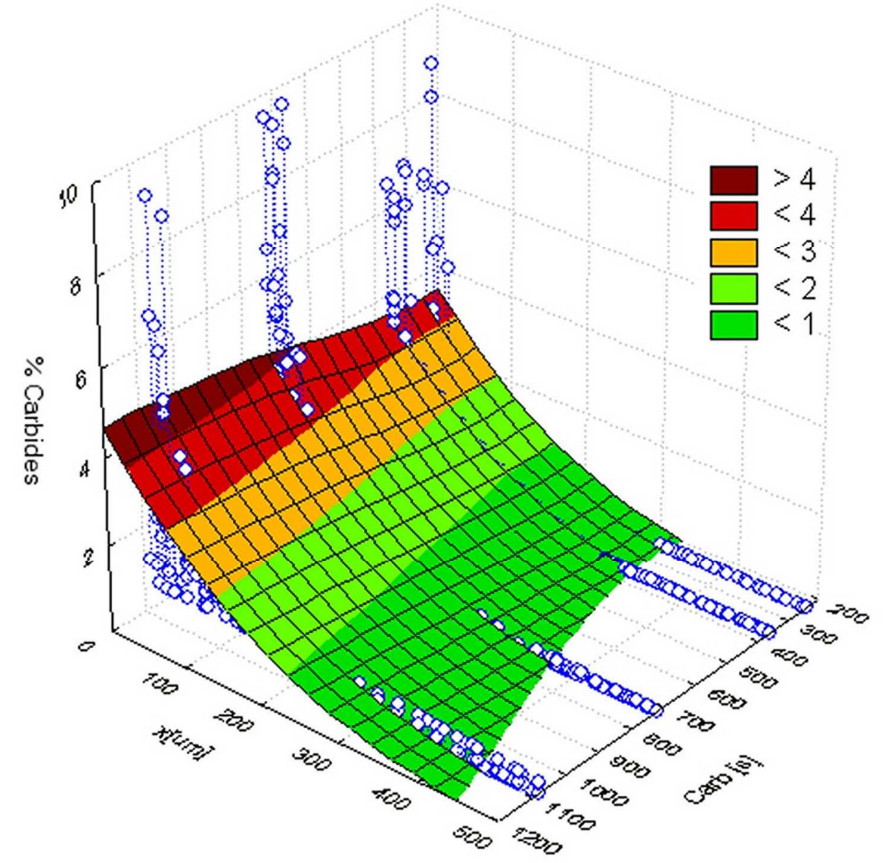

Fig. 1. Prediction of carbide content in the material (diagram showing relations of carbide content to distance from the surface and boost segment duration) 


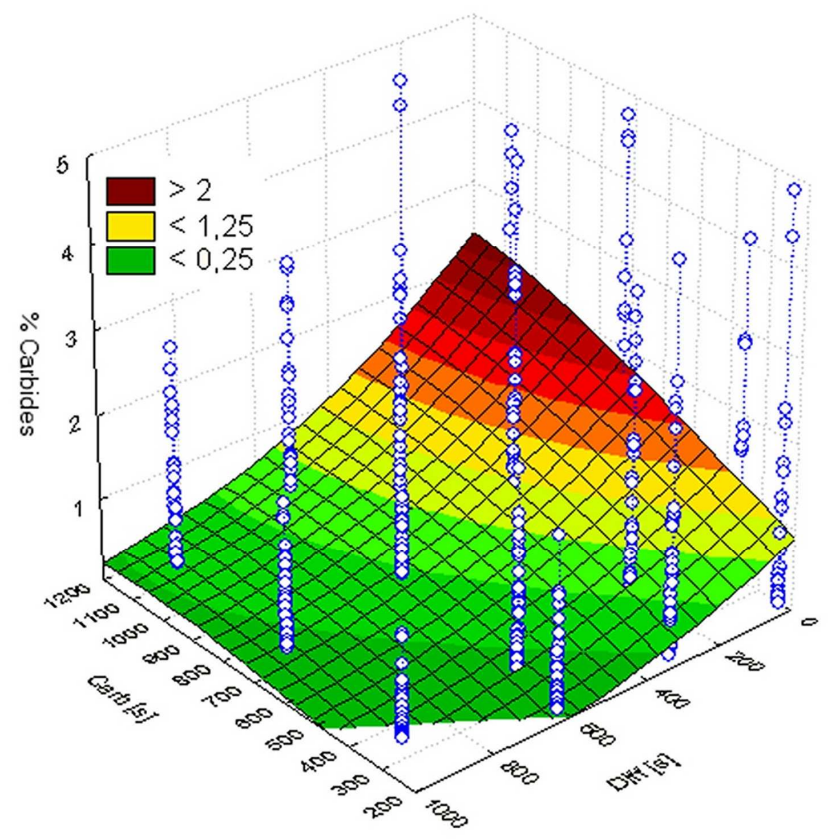

Fig. 2. Prediction of carbide content in the material (diagram showing relations of carbide content to boost segment time and diffusion segment time)

The characteristics of predictions for selected input parameters (distance from surface, boost segment time and diffusion segment time) were presented below (Fig. 1- Fig. 4). The graphs show the relation of a given output datum as a function of two selected parameters. It was assumed that all the remaining parameters, which are not included in the graph, had a constant value that was equal to the average value of learning cases. White spots were the network's responses to signals from learning sets.

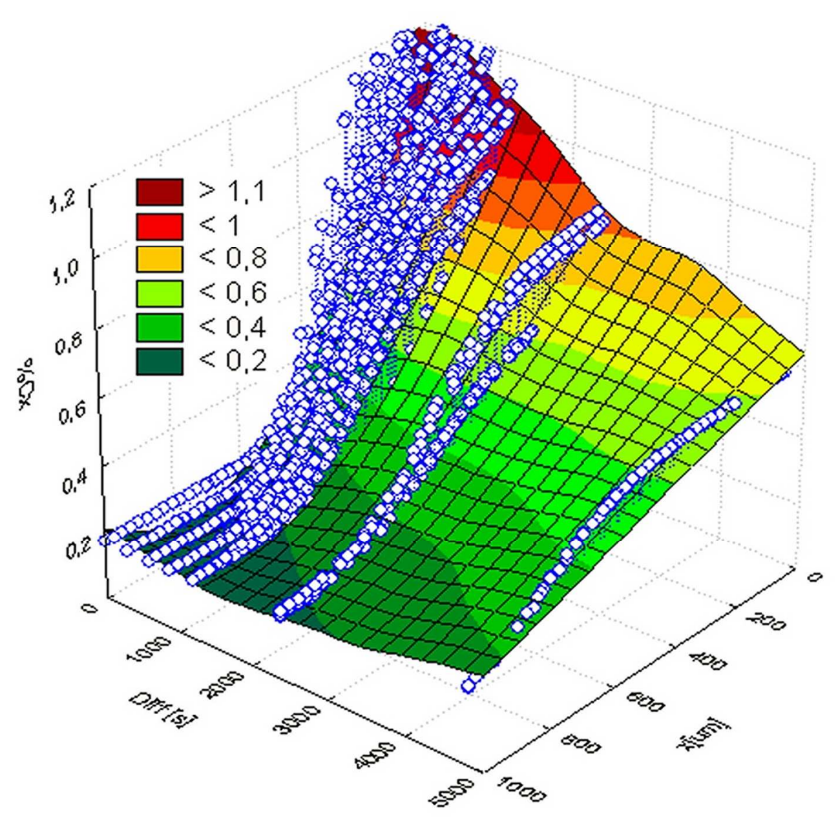

Fig. 3. Prediction of carbon concentration in the material (diagram showing relations of carbon concentration to distance from the surface and boost segment duration)

The biggest number of carbides are present near the surface of the material $(<10 \mu \mathrm{m})$, and their quantity decreases with distance from the surface. The shorter the boost segment, the smaller the thickness of carbides layer (Fig, 1, Fig. 2). 


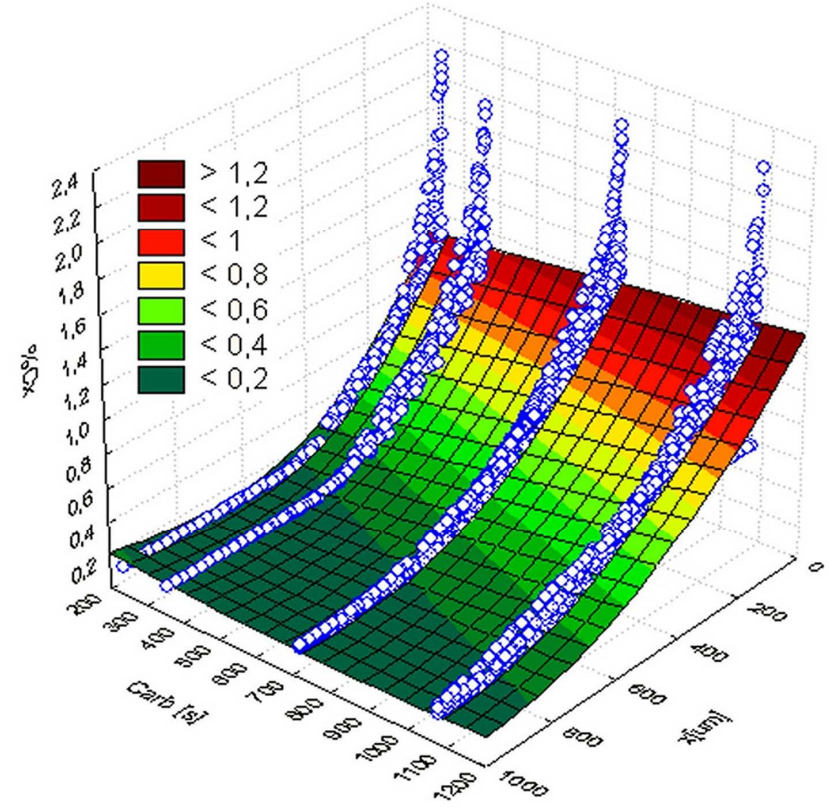

Fig. 4. Prediction of carbon concentration in the material (diagram showing relations of carbon concentration to distance from the surface and diffusion segment duration)

Carbon concentration in the material grows proportionally to the time of the boost segment. The highest concentration of carbon is present close to the surface and drops with the increase of distance from the surface. Moreover, in the case of short boost times (200-300 s), carbon profile is characterized by high steepness, which is reduced with the increase of boost time. In the graphs (Fig. 3), variable $x$ has a similar influence as in the figure (Fig. 4). It needs to be noted that short diffusion time means carbon accumulation in the subsurface layer of the material. Lengthening of the diffusion segment enables carbon transport deeper in the element.

Based on the qualitative assessments, we may say that a system of neural networks mapped the actual relations correctly. In the table (Tab. 1), the quality coefficient of the networks system is presented in relation to individual predicted values. In the table (Tab. 2), example answers provided by the neural network specifying carbon concentration in the material are gathered.

Table 1 Quality of neural networks to prediction carbide content and carbon concentration in material

\begin{tabular}{|l|c|c|}
\hline & Quality [\%MeC] & Quality [\%C] \\
\hline Training & 0.949 & 0.991 \\
\hline Testing & 0.925 & 0.990 \\
\hline Validation & 0.866 & 0.989 \\
\hline
\end{tabular}


Table 2 A juxtaposition of the responses for carbon output generated by the networks and those expected

\begin{tabular}{|l|c|c|c|}
\hline Sample & $\begin{array}{c}\text { Required } \\
\text { answer }[\% \mathbf{\%}]\end{array}$ & $\begin{array}{c}\text { Neural output } \\
{[\% \mathbf{\%}]}\end{array}$ & Difference [\%] \\
\hline Training & 0.505 & 0.476 & $\mathbf{0 . 0 2 9}$ \\
\hline Validation & 0.460 & 0.425 & $\mathbf{0 . 0 3 5}$ \\
\hline Testing & 0.427 & 0.379 & $\mathbf{0 . 0 4 8}$ \\
\hline Training & 0.393 & 0.339 & $\mathbf{0 . 0 5 4}$ \\
\hline Testing & 0.360 & 0.305 & $\mathbf{0 . 0 5 5}$ \\
\hline Validation & 0.336 & 0.277 & $\mathbf{0 . 0 5 9}$ \\
\hline Training & 0.311 & 0.258 & $\mathbf{0 . 0 5 3}$ \\
\hline Training & 0.287 & 0.237 & $\mathbf{0 . 0 5 0}$ \\
\hline Training & 0.263 & 0.226 & $\mathbf{0 . 0 3 7}$ \\
\hline Validation & 0.239 & 0.213 & $\mathbf{0 . 0 2 6}$ \\
\hline Training & 0.230 & 0.210 & $\mathbf{0 . 0 2 0}$ \\
\hline Training & 0.223 & 0.205 & $\mathbf{0 . 0 1 8}$ \\
\hline Training & 0.212 & 0.198 & $\mathbf{0 . 0 1 4}$ \\
\hline Testing & 0.202 & 0.193 & $\mathbf{0 . 0 0 9}$ \\
\hline Testing & 0.200 & 0.193 & $\mathbf{0 . 0 0 7}$ \\
\hline Training & 0.198 & 0.190 & $\mathbf{0 . 0 0 8}$ \\
\hline Validation & 0.196 & 0.187 & $\mathbf{0 . 0 0 9}$ \\
\hline Validation & 0.194 & 0.186 & $\mathbf{0 . 0 0 8}$ \\
\hline Training & 0.192 & 0.184 & $\mathbf{0 . 0 0 8}$ \\
\hline
\end{tabular}

\section{Discussion}

The figures (Fig. 1 - Fig. 4) clearly show the role of the boost and diffusion segments in the vacuum carburization process. The boost segment is in charge of carbon supply to the sample's surface, whereas the diffusion segment enables carbon transport deep in the material. A long boost stage and a short diffusion stage cause carbon accumulation (after exceeding the concentration threshold for carbon, carbide cluster precipitation occurs). A short boost stage and a long diffusion stage cause lowering and extension of the whole carbon profile. Prolongation of the diffusion stage, while keeping the same boost stage duration, causes extension of the carburized layer thickness (and simultaneously, a fall in carbon concentration on the surface). The observations are convergent with conclusions of other researches [16-19].

A system of artificial neural networks was used to model the described phenomena, although it was possible to solve this problem with a single neural network only. It needs to be explained that each properly trained network is able to correctly predict output variable values, but with the application of a single network, one output is preferred and the remaining may bring less accurate data. A system response of several networks for a single output will probably be not as precise as a specific network, but generally, it will predict all variables encumbered with less significant aggregate error.

\section{Conclusions}

The devised neural network correctly simulates the real relations and can be applied for the design of the vacuum carburizing treatment processes. The phenomenon of carbide formation and dissolution can also be simulated with an appropriate neural network, thus skipping the creation of a mathematical and physical standard. Unfortunately, when creating a precise simulator, it is necessary to carry out numerous experimental processes. Metallographic analysis is also time consuming and expensive. However, it should be stressed that this is a one-time effort.

The use of computer vacuum carburizing treatment simulators enables the design and optimization of industrial processes without actual technological trials. In practice, it means a reduction in the duration and cost of the treatment during mass production. 


\section{Acknowledgements}

Financial support of Structural Funds in the Operational Programme - Innovative Economy (IE OP) financed from the European Regional Development Fund - Project No POIG.0101.02-00-015/08 is gratefully acknowledged.

\section{References}

[1] E. El-Kassas, R. Mackie and A. El-Sheikh, A: Computers and Structures 79, (2001), pp. 1687.

[2] L. Dobrzanski and J. Trzaska: J. of Materials Processing Technology 155-156 (2004), pp. 1950.

[3] M. Raffiq, G. Bugmann and D. Easterbrook: Computers and Structures 79 (2001), pp. 1541.

[4] Z. Sterjovski, D. Nolan, K. Carpenter, D. Dunne and J. Norrish: J. of Materials Processing Technology 170 (2005), pp. 536.

[5] G. Cybenko: Continuous valued neural networks with two hidden layers are sufficient. Tech. rep., Tufts University, Medford, MA. Dept of Computer Science (1988).

[6] M. Hagan, H. Demuth and M. Beale: Neural Networks Design. Boston: PWS Publishing Company (1996).

[7] D. Rumelhart, G. Hinton and R. Wiliams: Learning internal representations by error propagation. Cambridge, MA: Explorations in the Microstructure of Cognition MIT Press (1986).

[8] S. Wang: Computers ind. Engineering Vol. 32, No. 1 (1997), pp. 67.

[9] K. Hornik, M. Stinchcombe and H. White: Neural Networks 115, Vol.2 (1989), pp. 359.

[10] P. Kula, R. Pietrasik, and K. Dybowski: Journal of Materials Processing Technology, 164-165 (2005), pp. 876.

[11] M. Korecki, P. Kula, and J. Olejnik: Industrial Heating, 3 (2011).

[12] S. Malinov and W. Sha: Computational Materials Science 28 (2003), pp. 179.

[13] F. Rosenblatt: Psychological Review 65 (1958), p. 386.

[14] D. Patterson: Artificial Neural Networks, Prentice Hall, Singapore, 1996.

[15] C. Bishop: Neural Networks for Pattern Recognition, Oxford University, 1995.

[16] M. Zhang and P. Kelly: Acta Materialia, Vol. 46, No. 13 (1998), pp. 4617.

[17] R. Heckel and H. Paxton: Trans. Am. Soc. Metals 53 (1961), pp. 539.

[18] H. Aaronson: Decomposition of austenite by diffusional processes, New York, 1962.

[19] A. Schneider and G. Inden: Computer Coupling Diagrams and Thermochemistry 31 (2007), pp. 141. 
Nanotechnology and Advanced Materials

10.4028/www.scientific.net/AMR.486

The Precipitation and Dissolution of Alloy Iron Carbides in Vacuum Carburization Processes for Automotive and Aircraft Applications - Part II

10.4028/www.scientific.net/AMR.486.303 